Helena von Eye Corletta ${ }^{1}$

Eunice Beatriz Martin Chaves ${ }^{2}$

MiRIam SigRUn KRaUSE ${ }^{3}$

EDISON CAPP ${ }^{4}$

\title{
Tratamento atual dos miomas
}

\author{
Current treatment of leiomyomas
}

Revisão

Palavras-chave

Mioma/terapia

Mioma/quimioterapia Leiomioma/quimioterapia

Leiomioma/cirurgia

Hormônio liberador de gonadotropina/

uso terapêutico

Receptores de progesterona/

uso terapêutico

Histerectomia

Embolização terapêutica

Keywords

Myoma/therapy

Myoma/drug therapy

Leiomyoma/drug therapy

Leiomyoma/surgery

Gonadotropin-releasing hormone/

therapeutic use

Receptors, Progesterone/

therapeutic use

Hysterectomy

Embolization, therapeutic

\section{Resumo}

Leiomiomas são tumores benignos. Eles surgem no miométrio e contêm quantidade variável de tecido conjuntivo fibroso. Cerca de $75 \%$ dos casos são assintomáticos, encontrados ocasionalmente durante exame abdominal, pélvico bimanual ou ultra-sonografia. Os sintomas são relacionados diretamente ao tamanho, ao número e à localização dos miomas. Nessa revisão, são apresentadas as abordagens terapêuticas atuais clínicas (anticoncepcionais orais, progestágenos e antiprogestágenos, análogos do hormônio liberador das gonadotrofinas (GnRH), e antiinflamatórios não esteróides) e cirúrgicas (histerectomia, miomectomia e embolização) para o tratamento de leiomiomas.

\section{Abstract}

Leiomyomas are benign tumors. They appear in the myometrium and present a variable amount of fibrous conjunctive tissue. About $75 \%$ of the cases are not symptomatic and are usually found during abdominal, bimanual pelvic examination or during ultrasonography. The symptoms are directly related to the size, number and localization of the myomas. In the present review, the current clinical therapeutic procedures (oral anti-conceptive drugs, progestins and anti-progestins, analogues of the gonadothrophins' releasing hormone $(\mathrm{GnRH})$, and non-steroid anti-inflammatory drugs), and also the surgical procedures (hysterectomy, myomectomy, embolization) are presented for the treatment of leiomyomas.
Correspondência:

Helena von Eve Corleto Rua Ramiro Barcelos, 910 cj. 905 CEP 90035-001 - Porto Alegre/RS Tel: (51) $3311-5699$ Fax: (51) 3311-6588 E-mail: hcorleta@portoweb.com.br

Recebido

18/04/2007

Aceito com modificacões
1 Professora do Departamento de Ginecologia e Obstetrícia e do Programa de Pós-Graduaccão em Medicina, Ciências Médicas, da Faculdade de Medicina da Universidade Federal do Rio Grande do Sul - UFRGS - Porto Alegre (RS), Brasil; Laboratório de Ginecologia e Obstetrícia Molecular do Centro de Pesquisas do Hospital de Clínicas de Porto Alegre - Porto Alegre (RS), Brasil; Gerar Centro de Reprodução Humana do Hospital Moinhos de Vento - Porto Alegre (RS), Brasil.

${ }^{2}$ Médica do Serviço de Ginecologia e Obstetrícia do Hospital de Clínicas de Porto Alegre - Porto Alegre (RS), Brasil.

${ }^{3}$ Médica Pesquisadora do Laboratório de Ginecologia e Obstetrícia Molecular do Centro de Pesquisas do Hospital de Clínicas de Porto Alegre - Porto Alegre (RS), Brasil; Universitaets-Fraven-Klinik Heidelberg, Abteilung Gynaekologische Endokrinologie und Fertilitaetsstoerungen, Heidelberg, Alemanha.

${ }^{4}$ Professor do Departamento de Ginecologia e Obstetrícia e do Programa de Pós-Graduação em Medicina, Ciências Médicas, da Faculdade de Medicina da Universidade Federal do Rio Grande do Sul - Porto Alegre (RS), Brasil; Laboratório de Ginecologia e Obstetrícia Molecular do Centro de Pesquisas do Hospital de Clínicas de Porto Alegre - Porto Alegre (RS), Brasil. 


\section{Introdução}

Leiomiomas são tumores benignos. Eles surgem no miométrio e contêm quantidade variável de tecido conjuntivo fibroso. Cerca de $75 \%$ dos casos são assintomáticos, encontrados ocasionalmente durante exame abdominal, pélvico bimanual ou ultra-sonografia. $\mathrm{O}$ sangramento uterino aumentado é a queixa mais comum, podendo levar a anemia.

Os sintomas são relacionados diretamente ao tamanho, ao número e à localização dos miomas. Os subserosos tendem a causar sintomas compressivos e distorção anatômica de órgãos adjacentes, os intramurais causam sangramento e dismenorréia, enquanto que os submucosos produzem sangramentos irregulares com maior freqüência. Além disso, observou-se que esses últimos estão mais associados à disfunção reprodutiva ${ }^{1-3}$. Extensa e recente revisão da literatura sugere que o leiomiossarcoma é uma lesão isolada; a transformação maligna dos miomas, se houver, é evento extremamente raro ${ }^{4}$.

O diagnóstico é baseado na história clínica (sinais e sintomas), no toque vaginal bimanual e na ultra-sonografia. A miomatose uterina é a causa mais comum de laparotomia em mulheres americanas, sendo responsável por 175.000 histerectomias e 20.000 miomectomias anu-

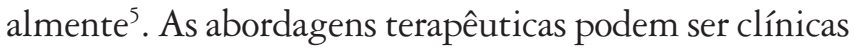
(anticoncepcionais orais, progestágenos e antiprogestágenos, análogos do hormônio liberador das gonadotrofinas (GnRH), e antiinflamatórios não esteróides) e cirúrgicas (histerectomia, miomectomia e embolização).

\section{Tratamento}

Mulheres com miomatose assintomática não necessitam tratamento, apenas acompanhamento e exame ginecológico de rotina, exceto aquelas com miomas muito volumosos ou que provoquem compressão ureteral ${ }^{6,7}$.

O tratamento das pacientes com miomas sintomáticos deve ser individualizado, levando-se em consideração a idade da paciente (proximidade da menopausa), o desejo de gestação, os sintomas provocados, o tamanho e a localização dos miomas (Figura 1$)^{8}$. O objetivo do tratamento clínico é o alívio dos sintomas. Como a grande maioria das pacientes com miomatose torna-se assintomática após a menopausa, o tratamento medicamentoso pode tornar os sintomas aceitáveis até a chegada da menopausa, evitandose os riscos associados aos tratamentos cirúrgicos.

\section{Tratamento Clínico}

\section{Anticoncepcionais orais}

Quanto aos contraceptivos orais, não há evidência de que sejam efetivos no tratamento de miomas; no entanto, são eficazes para correção do sangramento uterino disfuncional ${ }^{7,9}$.

\section{Progestágenos e antiprogestágenos}

Em função de seu baixo custo e facilidade de administração, são utilizados no tratamento dos distúrbios menstruais disfuncionais, muitas vezes concomitantes à miomatose. Os derivados da 19-norprogesterona



Figura 1 - Proposta de algoritmo para tratamento dos miomas considerando a situação clínica e as alternativas atuais. 
apresentam maior efeito antiestrogênico e menor efeito androgênico, trazendo melhora no caso da menometrorragia, tanto quando usados na segunda fase do ciclo, como de forma contínua. O acetato de medroxiprogesterona $150 \mathrm{mg}$, intramuscular a cada três meses, também é bastante utilizado por causar amenorréia e melhorar a anemia ${ }^{10}$.

Os progestágenos não são utilizados para diminuir o volume dos miomas. Existem evidências, inclusive, de haver aumento no número e tamanho dos miomas com esta medicação ${ }^{2,11}$. Agentes como mifepristone, que antogonizam o efeito dos progestágenos nos seus receptores, diminuem o volume dos miomas de forma similar à dos agonistas do $\mathrm{GnRH}^{12}$. Em trabalho recente de Eisinger et al. ${ }^{13}$, o mifepristone (RU486), em doses de 5 a $10 \mathrm{mg} /$ dia durante um ano, reduziu em aproximadamente $50 \%$ o volume uterino total e, diferentemente de outras drogas, a redução se manteve em $42 \%$ após cinco meses da suspensão do tratamento ${ }^{13}$.

O DIU com levonorgestrel tem efeito benéfico no sangramento por miomatose; entretanto, não há redução do volume dos miomas ${ }^{14,15}$.

Análogos do hormônio liberador das gonadotrofinas

Os análogos do GnRH são medicações efetivas no tratamento clínico, levando à redução de 35-60\% do volume dos miomas em três meses. Normalmente, são utilizados no preparo cirúrgico das pacientes, pois, em função dos efeitos colaterais, como perda de massa óssea, distúrbio do perfil lipídico e sintomas climatéricos, não devem ser usados por mais de seis meses. Caso não seja realizada a cirurgia, a interrupção do tratamento com análogos leva ao reaparecimento da doença; por isto, os análogos do GnRH são utilizados primariamente para contemporizar e permitir a recuperação do hematócrito antes da cirurgia. Entretanto, como a suplementação de ferro isolado traz beneficio para um número significativo de pacientes, os custos, os efeitos adversos e a eficácia devem ser avaliados quando se propõe o uso do GnRH no pré-operatório ${ }^{7,8,10}$.

\section{Antiinflamatórios não esteróides}

Estes fármacos são utilizados no tratamento do sangramento vaginal excessivo e dismenorréia; entretanto, não parecem reduzir as perdas sangüíneas em mulheres com miomatose $\mathrm{e}^{10,16}$.

Cirurgia

\section{Histerectomia}

O tratamento definitivo da miomatose sintomática é cirúrgico ${ }^{8,10}$. São indicações de histerectomia:
1. presença de sintomas;

2. falha no tratamento clínico associado a sangramento uterino anormal, com prole constituída ou sem desejo de gestação.

Miomas grandes assintomáticos provavelmente podem ficar sem tratamento, pois o risco do tratamento de um leiomiossarcoma é menor do que a mortalidade pela histerectomia ${ }^{4,5,17}$.

As pacientes que optam pela histerectomia relatam melhora na qualidade de vida e têm índices de satisfação melhores do que em outros tratamentos ${ }^{18}$. Recente revisão da biblioteca Cochrane avaliou as diferentes formas de histerectomia realizadas para tratamento de doença benigna do útero: histerectomia abdominal (HA), histerectomia vaginal (HV) e histerectomia videolaparoscópica (VLPC) que pode ser realizada totalmente pela via laparoscópica ou ser assistida pela via vaginal ${ }^{19}$. Como resultado, a $\mathrm{HV}$, em comparação à $\mathrm{HA}$, demonstrou ter menor tempo de internação hospitalar, menor tempo para retorno às atividades laborais e menor incidência de infecções. A comparação da abordagem VLPC à HA favoreceu a primeira em tempo de internação hospitalar, retorno ao trabalho e menos episódios febris; no entanto, na VLPC houve significativamente mais lesão do trato urinário. A histerectomia VLPC não se mostrou superior à HV. Nesta revisão sistemática os estudos foram heterogêneos e aspectos de complicações e resultados a médio e longo prazo não foram contemplados.

A escolha da melhor abordagem para histerectomia baseia-se em diversos fatores, incluindo: características clínicas das pacientes, úteros com até $500 \mathrm{~cm}^{3}$, habilidade e experiência do cirurgião, técnica de maceração dos miomas, e morbidade e custo associados com o procedimento ${ }^{7,20}$. A mortalidade total por histerectomia é de $0,5-2$ por 1.000 , e em 1,5 a $2 \%$ dos casos ocorrem lesões viscerais. Deve ser lembrado que as complicações cirúrgicas são mais comuns nas mulheres operadas por miomatose e que diminuem com a idade da paciente.

Outra discussão ainda remanescente é da histerectomia total versus subtotal. Esta última, por deixar o colo uterino, é muito mais rápida e têm significativamente menos complicações. Na década de 80, esta técnica ganhou muitos adeptos, após a publicação de Kilkku, que demonstrou menores taxas de dispareunia após a histerectomia subtotal em comparação à total ${ }^{21}$. Em $2002^{22}$, ensaio clínico randomizado avaliou que as duas técnicas tinham resultados similares quanto a todas as funções pélvicas (urinária, evacuatória e 
sexual). A histerectomia subtotal apresentou melhor recuperação a curto prazo e menos intercorrências febris de curto prazo; entretanto, mais sangramento (devido a endométrio residual) e prolapso cervical ${ }^{22}$. Gupta e Manyonda ${ }^{18}$ argumentam que a histerectomia subtotal, nesta época de freqüentes litígios contra os médicos, continuará sendo realizada. Acreditamos que a prevalência de carcinoma de colo uterino e a baixa cobertura do rastreamento em nosso país devam ser levadas em consideração na tomada de decisão quanto à histerectomia total ou subtotal.

\section{Miomectomia}

A indicação de miomectomia dependerá do desejo da paciente de manter a fertilidade e o útero. É importante ressaltar que a recorrência de miomas é estimada entre $15-30 \%$, sendo que $10 \%$ das mulheres necessitam uma nova intervenção ${ }^{6,7}$. A miomectomia pode ser laparotômica, por via vaginal, laparoscópica ou histeroscópica, dependendo da localização, do tamanho e do número de miomas a serem retirados ${ }^{12}$. As complicações relacionadas ao procedimento aumentam com o número de miomas. O risco de recorrência é menor quando apenas um mioma está presente e é retirado $^{7}$. Miomas protruindo pelo orifício cervical externo são retirados por via vaginal.

Histeroscopia atualmente é a melhor forma de abordagem dos miomas submucosos ${ }^{8,23}$. A remoção de miomas submucosos pediculados é relativamente fácil. Os submucosos com componente intramural ou os intramurais com compressão do endométrio e distantes da serosa também podem ser abordados por via histeroscópica, necessitando ocasionalmente mais de uma intervenção ${ }^{16}$.

\section{Embolização}

A embolização da artéria uterina (EAU) tem sido utilizada para tratar uma série de problemas hemorrágicos em ginecologia e obstetrícia, sendo também uma opção conservadora para pacientes com miomas sintomáticos que têm contra-indicação ou não desejam se submeter aos riscos cirúrgicos ${ }^{12,24}$. A embolização da artéria uterina causa infarto do mioma, reduzindo seu tamanho em $50 \%$ e a sintomatologia em $85 \%$ dos $\operatorname{casos}^{25,26}$.
Edwards et al. ${ }^{24}$, em ensaio clínico randomizado recentemente publicado, compararam histerectomia à embolização de miomas sintomáticos. Os resultados demonstraram que as pacientes que realizaram EAU tiveram alta hospitalar significativamente mais cedo do que as submetidas à histerectomia. Entretanto, o grau de satisfação um ano após os procedimentos não diferiu entre os grupos. A longo prazo, as pacientes com EAU tiveram mais intercorrências e, em $10 \%$ delas, foi realizada histerectomia posteriormente.

As indicações para EAU não estão claramente definidas. Mais estudos são necessários para determinar qual localização, o tamanho e o número de miomas ideal para melhor resposta a esta terapêutica. Ainda não existem dados suficientes para concluir que a EAU é um procedimento seguro para mulheres que desejam preservar a fertilidade. Mesmo que alguns nascimentos tenham sido relatados, a incidência de trabalho de parto prematuro, abortamento e sangramento e a taxa de gestação não foram adequadamente estudadas $^{27,28}$.

As complicações associadas ao procedimento incluem dor abdominal, febrícula, infecção, expulsão do mioma, necessidade de histerectomia após o procedimento (1 a 2\%) e mortalidade de 0,1 a 0,2 por 1000 procedimentos $^{8,20,28}$.

\section{Infertilidade}

Os miomas estão associados à infertilidade em 5 a $10 \%$ dos casos. Entretanto, quando todas as causas de infertilidade são excluídas, os miomas são responsáveis por apenas 2 a $3 \%$ dos $\operatorname{casos}^{29}$.

Estudo prospectivo com 46 mulheres com miomas que realizaram fertilização in vitro com transferência de embriões mostrou taxas de gestação e implantação semelhantes a um grupo de 50 mulheres sem miomatose uterina, quando os miomas não distorciam a cavidade endometrial ${ }^{5}$.

Pacientes com dificuldade de gestação e miomatose devem inicialmente realizar toda a investigação para infertilidade ${ }^{29}$. Devem ser tratadas as pacientes inférteis com alteração da cavidade endometrial ${ }^{30}$. Alguns autores recomendam tratamento de miomas intramurais maiores que $7 \mathrm{~cm}$ (23); entretanto, isto persiste controverso ${ }^{30}$, devendo ser individualizados 5 . 
1. Wegienka G, Baird DD, Hertz-Picciotto I, Harlow SD, Hartmann $\mathrm{KE}$. Uterine leiomyomata (fibroids): are bleeding symptoms more likely to be reported after diagnosis? J Clin Epidemiol. 2004;57(3):3 18-20.

2. Flake GP, Andersen J, Dixon D. Etiology and pathogenesis of uterine leiomyomas: a review. Environ Health Perspect. 2003; 111 (8):1037-54.

3. Lippman SA, Warner M, Samuels S, Olive D, Vercellini P, Eskenazi B. Uterine fibroids and gynecologic pain symptoms in a populationbased study. Fertil Steril. 2003;80(6):1488-94.

4. Schwartz PE, Kelly MG. Malignant transformation of myomas: myth or reality? Obstet Gynecol Clin North Am. 2006;33(1): 183 98, xii.

5. The Practice Committee of the American Society for Reproductive Medicine. Myomas and reproductive function. Fertil Steril. 2006;86(5 Suppl):S194-9.

6. Guarnaccia MM, Rein MS. Traditional surgical approaches to uterine fibroids: abdominal myomectomy and hysterectomy. Clin Obstet Gynecol. 2001;44(2):385-400.

7. Stewart EA. Uterine fibroids. Lancet. 2001;357(9252):293-8.

8. Lefebvre G, Vilos G, Allaire C, Jeffrey J, Arneja J, Birch C, et al. The management of uterine leiomyomas. J Obstet Gynaecol Can. 2003;25(5):396-418; quiz 9-22.

9. Carr BR, Marshburn PB, Weatherall PT, Bradshaw KD, Breslau NA, Byrd W, et al. An evaluation of the effect of gonadotropin-releasing hormone analogs and medroxyprogesterone acetate on uterine leiomyomata volume by magnetic resonance imaging: a prospective, randomized, double blind, placebo-controlled, crossover trial. J Clin Endocrinol Metab. 1993;76(5):1217-23.

10. Lethaby A, Vollenhoven B. Fibroids (uterine myomatosis, leiomyomas). Clin Evid. 2002(7): 1666-78.

11. De Leo V, la Marca A, Morgante G, Severi FM, Petraglia F. Administration of somatostatin analogue reduces uterine and myoma volume in women with uterine leiomyomata. Fertil Steril. $2001 ; 75(3): 632-3$.

12. Hurst BS, Matthews ML, Marshburn PB. Laparoscopic myomectomy for symptomatic uterine myomas. Fertil Steril. 2005;83(1):1-23.

13. Eisinger SH, Bonfiglio T, Fiscella K, Meldrum S, Guzick DS. Twelvemonth safety and efficacy of low-dose mifepristone for uterine myomas. J Minim Invasive Gynecol. 2005; 12(3):227-33.

14. Magalhaes J, Aldrighi JM, de Lima GR. Uterine volume and menstrual patterns in users of the levonorgestrel-releasing intrauterine system with idiopathic menorrhagia or menorrhagia due to leiomyomas. Contraception. 2007;75(3):193-8.

15. Maruo T, Oharaa N, Matsuo H, Xua Q, Chena W, Sitruk-Wareb $\mathrm{R}$, et al. Effects of levonorgestrel-releasing IUS and progesterone receptor modulator PRM CDB-2914 on uterine leiomyomas.
Contraception. 2007; in press, corrected proof, available online 21 March

16. Banu NS, Manyonda IT. Alternative medical and surgical options to hysterectomy. Best Pract Res Clin Obstet Gynaecol. 2005; 19(3):431-49.

17. The Practice Committee of the American Society for Reproductive Medicin. Myomas and reproductive function. Fertil Steril. 2004;82 Suppl 1:S111-6.

18. Gupta S, Manyonda I. Hysterectomy for benign gynaecological disease. Current Obstetrics \& Gynaecology. 2006;16(3): 147-53.

19. Johnson N, Barlow D, Lethaby A, Tavender E, Curr E, Garry R. Surgical approach to hysterectomy for benign gynaecological disease. Cochrane Database Syst Rev. 2006(2):CD003677.

20. Manyonda I, Sinthamoney E, Belli AM. Controversies and challenges in the modern management of uterine fibroids. Bjog. 2004; 111 (2):95-102.

21. Kilkku P. Supravaginal uterine amputation vs. hysterectomy. Effects on coital frequency and dyspareunia. Acta Obstet Gynecol Scand. 1983;62(2):141-5.

22. Thakar R, Ayers S, Clarkson P, Stanton S, Manyonda I. Outcomes after total versus subtotal abdominal hysterectomy. N Engl J Med. 2002;347(17):1318-25.

23. Pritts EA. Fibroids and infertility: a systematic review of the evidence. Obstet Gynecol Surv. 2001;56(8):483-91.

24. Edwards RD, Moss JG, Lumsden MA, Wu O, Murray LS, Twaddle $S$, et al. Uterine-artery embolization versus surgery for symptomatic uterine fibroids. N Engl J Med. 2007;356(4):360-70.

25. Gupta JK, Sinha AS, Lumsden MA, Hickey M. Uterine artery embolization for symptomatic uterine fibroids. Cochrane Database Syst Rev. 2006(1):CD005073.

26. Goodwin SC, Bonilla SM, Sacks D, Reed RA, Spies JB, Landow WJ, et al. Reporting standards for uterine artery embolization for the treatment of uterine leiomyomata. J Vasc Interv Radiol. 2001;12(9): 1011-20.

27. ACOG. Committee Opinion. Uterine artery embolization. Obstet Gynecol. 2004;103(2):403-4.

28. Marshburn PB, Matthews ML, Hurst BS. Uterine artery embolization as a treatment option for uterine myomas. Obstet Gynecol Clin North Am. 2006;33(1):125-44.

29. Silva ALBd, Seibel SA, Capp E, Corleta HvE. Miomas e infertilidade: bases fisiopatológicas e implicações terapêuticas. Revista Brasileira de Saúde Materno Infantil. 2005;5:13-8.

30. Surrey ES. Impact of intramural leiomyomata on in-vitro fertilizationembryo transfer cycle outcome. Curr Opin Obstet Gynecol. 2003;15(3):239-42. 